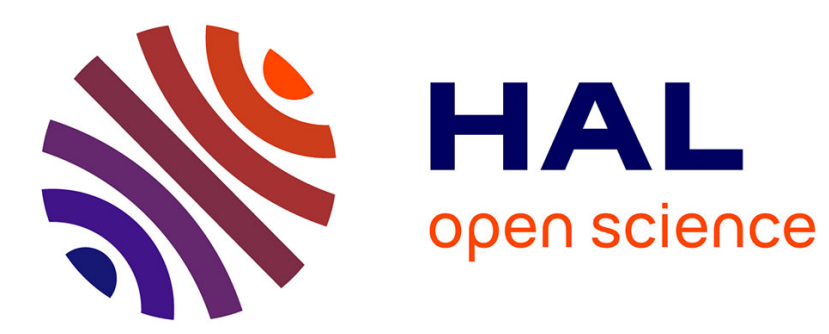

\title{
A design methodology for delta-sigma converters based on solid-state passive filters \\ Philippe Benabes
}

\section{To cite this version:}

Philippe Benabes. A design methodology for delta-sigma converters based on solid-state passive filters. 2013 IEEE 11th International New Circuits and Systems Conference (NEWCAS 2013), Jun 2013, Paris, France. pp.1-4, 10.1109/NEWCAS.2013.6573617 . hal-00862354

\section{HAL Id: hal-00862354 \\ https://hal-centralesupelec.archives-ouvertes.fr/hal-00862354}

Submitted on 16 Sep 2013

HAL is a multi-disciplinary open access archive for the deposit and dissemination of scientific research documents, whether they are published or not. The documents may come from teaching and research institutions in France or abroad, or from public or private research centers.
L'archive ouverte pluridisciplinaire HAL, est destinée au dépôt et à la diffusion de documents scientifiques de niveau recherche, publiés ou non, émanant des établissements d'enseignement et de recherche français ou étrangers, des laboratoires publics ou privés. 


\title{
A design methodology for delta-sigma converters based on solid-state passive filters
}

\author{
Philippe BENABES \\ Department of Signal and Electrical Systems, \\ Supelec \\ Gif/Yvette, France \\ Email: philippe.benabes@ @upelec.fr
}

\begin{abstract}
In the context in the ENIAC ARTEMOS project for the design of agile radio front ends, this paper shows a methodology for the design of agile bandpass continuous-time delta sigma based on acoustic tunable resonators. These resonators use BST materials which have the property to be tunable by an external voltage, allowing changing the resonance frequency of filters by a few percent. Using such filters, the Oversampling ratio of delta sigma may decrease as the central frequency of the modulator may be adjusted precisely, allowing the change the band without having to acquire the whole spectrum. Direct RF applications at $2.4 \mathrm{GHz}$ are expected within this project.
\end{abstract}

\section{INTRODUCTION}

In the context of software-defined radio, bandpass continuous-time (BPCT) delta-sigma $(\Delta \Sigma)$ modulators are good candidates for direct conversion of radio-frequency signals. One possibility is to use wideband conversion. The channel choice is fully performed by software, but this choice induces very high sampling frequencies due to the OSR required by the modulator. Another choice is to convert with a smaller bandwidth, but the central frequency should be adjustable. One of the bottlenecks is the sensitivity to the resonance frequency of the CT filters used in the loop of the modulator. Ideally the central frequency should be extremely precise and adjustable in order to be able to choose to the expected channel. Classical passive filters are too much sensitive to temperature and process dispersion, but new materials such as BST $\left(\mathrm{Ba}_{1-\mathrm{x}} \mathrm{Sr}_{\mathrm{x}} \mathrm{TiO}_{3}\right)$ [1] offer very stable and adjustable resonance frequencies, and become good candidates for the design of bandpass $\Delta \Sigma$ modulators. Yet, passive filters based on these materials have parasitic resonances which cannot be removed. Previous work using SAW resonators as loop filters has been presented by $\mathrm{Yu}$ and $\mathrm{Xu}$ in 2007 [2], but they take into account only the main resonance but not the harmonic ones. The methodology for the design of bandpass modulators based on such filters should then be adapted accordingly.

The second section of this paper presents some results, simulations and modeling of the BST materials. The third section of this paper deals with a methodology for the design

This work is part of the ENIAC ARTEMOS project (Agile RF Transceivers and Front-Ends for Future Smart Multi-Standard Communications Applications) of BPCT $-\Delta \Sigma$ modulators using BST filters, and the fourth section will show some simulation results of the output of a BST-based second-order modulator.

\section{THE BST FILTERS}

\section{A. Presentation of the BST material}

A technology for tunable BST acoustic components is being developed by CEA-LETI in France. These components behave as resonators with a resonance frequency that can be controlled by an external voltage.

These resonators use $\mathrm{Ba}_{\mathrm{x}} \mathrm{Sr}_{1-\mathrm{x}} \mathrm{TiO} 3$ (BST) [1] as the active material which acts both as the transducer material (to generate the acoustic waves causing the resonance to appear) and the source of tuneability in this resonator. In the implementation developed within the ARTEMOS project [3], a thin BST film is sandwiched between two electrodes and is located above an acoustic Bragg mirror so as to prevent leakage of acoustic waves into the substrate and therefore to obtain a sharp resonance. Figure 1. shows a cross section of such a resonator.

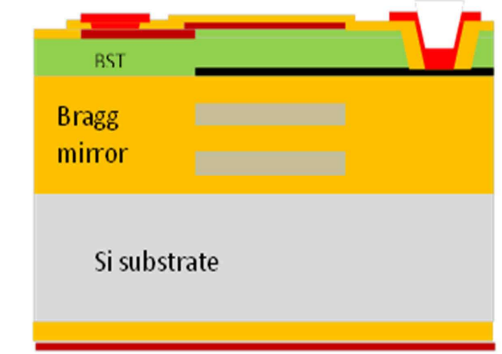

Figure 1. Schematic cross section of a BST resonator

Depending on the DC voltage applied to the resonator, the resonance frequency can vary by a few percent.

\section{B. Modeling the BST filters}

In the context of the ARTEMOS project, new resonators are being developed, with an expected central frequency of 2.4Ghz. Figure 3 illustrates the dependence of the impedance on the DC voltage. It can be seen that the resonance frequency 
is more sensitive to the DC voltage that the anti-resonance frequency. With this updated process flow, we expect relative frequency shifts induced by the application of a DC bias to reach $6 \%$ for the resonance frequency and $3 \%$ for the antiresonance frequency.

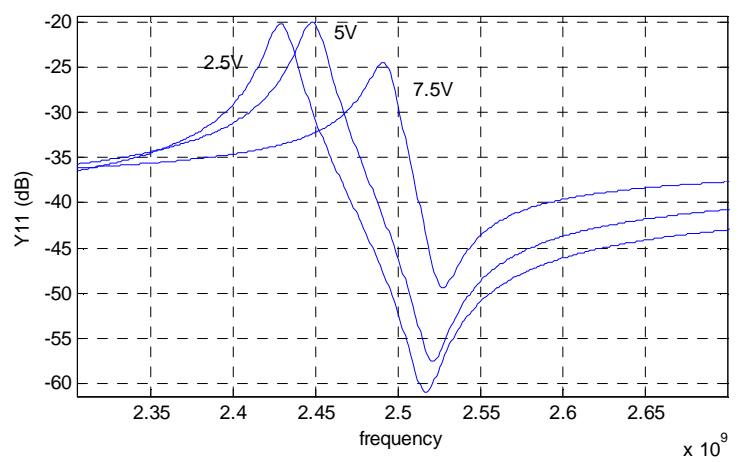

Figure 2. Simulated admitance of the BST filters developed in the ARTEMOS project, for different bias voltages

\section{Design of a resonator based on a BST filter}

The anti-resonance, which is due to the parallel parasitic capacitance between the active part and the ground, has to be canceled. This can be done by adding a parallel capacitance driven by the opposite voltage [2]. The best resulting scheme is to use a differential filter with two BST resonators and two compensation capacitances as seen in Figure 3.

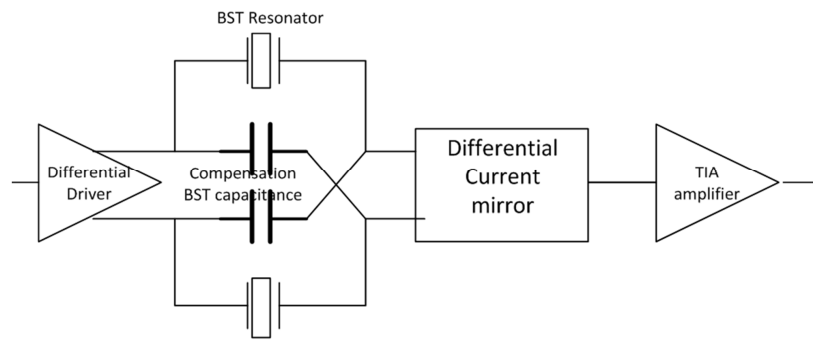

Figure 3. Parasitic capacitance compensation circuit.

In this schematic, the BST filter is driven by a voltage source. The resulting current is transformed into a voltage via a transimpedance amplifier. The serial impedance at resonance of the BST filters is quite small (a few tens of Ohms). Consequently, the output impedance of the drivers and the input impedance of the TIA should be as small. As it is quite difficult to design a stable TIA amplifier with very small input impedance, a differential current mirror is added. The input impedance of this mirror can be lowered without affecting its stability.

This principle can be modeled, as shown in Erreur ! Source du renvoi introuvable.

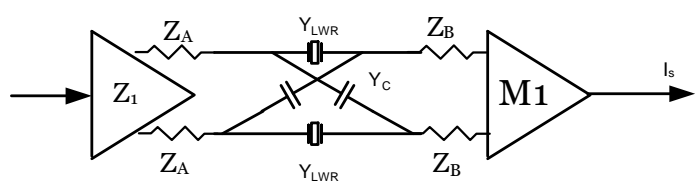

Figure 4. Model of the resonator

The admittance of the BST filter is denoted $Y_{L W R}$, the admittance of the compensation capacitance is $Y_{C}$, The voltage driver is denoted by $\mathrm{Z} 1$ and its output impedance by $Z_{A}$; the current mirror is $M 1$ and its input impedance is denoted $Z_{B}$.

After simplification, by supposing that matching is perfect between the two branches, the output current is:

$$
I_{S}=\frac{Y_{L W R}-Y_{C}}{1+\left(Y_{L W R}+Y_{C}\right)\left(Z_{A}+Z_{B}\right)+4 Y_{L W R} Y_{C} Z_{A} Z_{B}} V_{e}
$$

If $\mathrm{Z}_{\mathrm{A}}$ and $\mathrm{Z}_{\mathrm{B}}$ were zero, the transfer function would be ideally $Y_{L W R}-Y_{C}$. But the effective non-zero values modify the transfer function. The different active elements $\left(Z_{1}\right.$ and $\mathrm{M}_{1}$ ) will also act as lowpass filters.

\section{Model of the whole filter}

The BST resonator characteristics have been evaluated using a finite element modeling software. After applying the capacitance compensation technique presented in [2], the admittance shown in Fig. 6 is obtained (frequency is normalized to the sampling frequency).

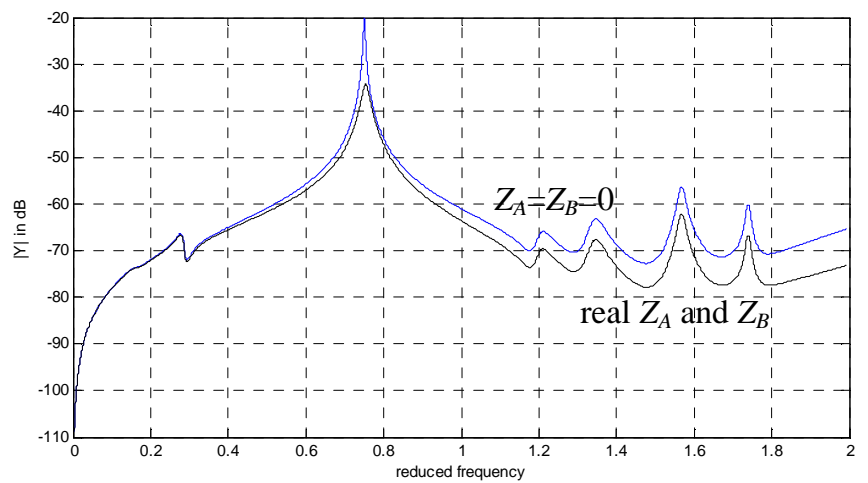

Figure 5. BST admitance after capacitance compensation technique, with and without taking into account $\mathrm{ZA}$ and $\mathrm{ZB}$.

The impedances of the driver and the mirror affect the filter response: they reduce the quality factor and behave as a lowpass filter, which attenuates the high-frequency components. The gain losses will have to be compensated for in the rest of the loop.

As the filter transfer function is not an ideal resonator but also presents harmonic resonances, a new methodology for the design of BPCT $\Delta \Sigma$ modulators has be proposed.

\section{DESIGN OF A DELTA SIGMA MODULATOR BASED ON A BST FILTER}

The methodology for designing a $\Delta \Sigma$ converter consists in starting from the available individual filters (the BST resonators), and deduce from their characteristics an adapted $\Delta \Sigma$ topology.

All acoustic waves' filters are based on individual resonators. The transfer function close to the resonance frequency can be modeled as 


$$
\frac{\omega_{0} s-a}{s^{2}+\frac{\omega_{0} s}{Q}+\omega_{0}^{2}}
$$

where the phase at resonance frequency has been accounted for by introducing a zero in the numerator of the transfer function. This model is of course valid in a small frequency range around the resonance frequency. But it is a good starting point for an optimization algorithm.

Based on previous work [4] on $\Delta \Sigma$ topologies, we propose to use the following methodology:

- Start from a known noise transfer function (NTF), capable of meeting the expected specifications in discrete-time

- Use a discrete-time to continuous-time transform to get the global CT main modulator filter transfer function

Optimize the loop delay so that the loop filter has the same phase as the real filter

- Deduce a topology based upon ideal resonators

- Design separately each filter and deduce its characteristics and imperfections

- $\quad$ Replace the ideal filters by their real model

- Adjust manually (if necessary) the coefficients values, if the modulator characteristics have changed too much with the real filter

\section{A. Initial discrete-time transfer-function}

The discrete-time transfer function of a $\Delta \Sigma$ modulator can be obtained by several methods. One of them is based on Richard Schreier's Matlab toolbox [5].

Unfortunately, this tool does not take into account the quality factor of the analog filters. A tool has been developed by the author, based on the methodology published in [6]:

The NTF of a modulator of order $2 m$ can be expressed as the product of $m$ second-order NTFs

$$
\begin{aligned}
& \operatorname{NTF}(z)=\prod_{l=1}^{m} N T F_{l}(z) \\
& \text { where } \operatorname{NTF}_{l}(z)=\frac{1}{1+c_{l} D_{l}(z)}
\end{aligned}
$$

$D_{l}(z)$ is the transfer function of a second-order resonator, and can be expressed by :

$$
D_{l}(z)=\frac{\alpha p_{l} z^{-1}-z^{-2}}{1-p_{l}\left(\frac{Q_{l}-2 \pi f_{l}}{Q_{l}-\pi f_{l}}\right) z^{-1}+\left(1-\frac{2 \pi f_{l}}{Q_{l}}\right) z^{-2}}
$$

where $p_{l}=2 \cos \left(2 \pi \frac{F_{l}}{F_{s}}\right), Q_{i}$ is the quality factor of the expected CT filter, and coefficients $\alpha$ can be chosen equal to $0.5[4]$.
Finally, the global DT filter transfer function $D(z)$ can be expressed as:

$$
D(z)=\left(\prod_{l=1}^{m}\left(1+c_{l} D_{l}(z)\right)\right)-1
$$

\section{B. From discrete-time to continuous-time}

In order to obtain the equivalent CT transfer function, a transformation $p<->z$ is required. The impulse invariance transform gives an exact response at the sampling times [7]. It has been shown that the CT filter transfer function $G(s)$ can be found by resolving equation (6):

$$
\begin{aligned}
& F(z)=\left(1-z^{-1}\right) \mathrm{Z}_{T}\left\{L^{-1}\left[\frac{G(s)}{s} e^{-d s}\right]\right\}-D(z), \\
& \text { with } D(z)=\sum_{k} a_{k} z^{-k}
\end{aligned}
$$

where $L$ stands for the Laplace transform, $Z_{T}$ the $Z$ transform after sampling at period $T$, and $d$ is the delay introduced by the $\mathrm{ADC}$ and the DAC. The term $D(z)$ has to be introduced when the delay $d$ is non-zero, to be able to solve this equation [6]. The order of $D(z)$ is the integer part of the delay (normalized to the sampling frequency) plus 1 .

This equation results into a modified topology as shown in Figure 6. The term $D(z)$ can be implemented as a direct feedback from the DAC output to the ADC input for the terms of $D(z)$ which are longer that the DAC delay $\left(D_{2}(z)\right)$. The first terms can be implemented as an analog feedback $\left(D_{l}(z)\right)$ as seen in Figure 7. (At this time $F(s)=1$ )

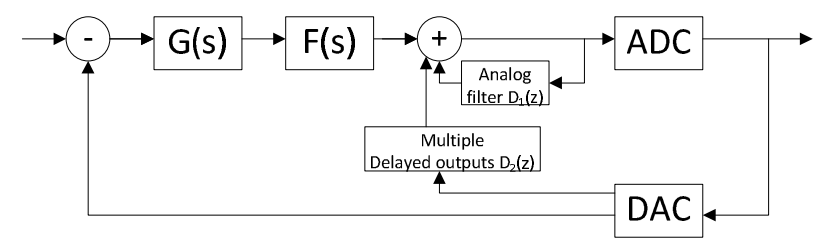

Figure 6. CT topology with delay

The loop delay has to be adjusted. By solving equation (6) for several delays, it can be seen that the zero of $G(p)$ depends directly on the loop delay $d$. Therefore the loop delay can be adjusted in an optimization loop in order to get the expected zero.

\section{Introducing a lowpass filter}

It was seen in section II that due to electrical realization constraints, the filter does not behave as a 'pure' resonator, but all electrical elements behave as lowpass filters and introduce new poles in the loop. These poles can be taken into account during the design of the modulator and compensated for.

Any filter transfer function $G(s)$ can be decomposed as the product of a filter $G^{\prime}(s)$ by $F(s)$ plus an additive term $F_{c}(s)$, where $F(s)$ is an arbitrary filter. The poles of $G$ ' are the same as those of $G$, and the poles of $F_{C}$ are the same as those of $F$.

This can be expressed as: 


$$
\frac{G_{n}(s)}{G_{d}(s)}=\frac{G_{n}^{\prime}(s)}{G_{d}(s)} \frac{F_{n}(s)}{F_{d}(s)}+\frac{F c_{n}(s)}{F_{d}(s)}
$$

$G(s)=G_{n}(s) / G_{d}(s)$ is the expected transfer function, $F(s)$ $F_{n}(s) / F_{d}(s)$ is the filtering introduced by electronics components, $G^{\prime}(s)=G_{n}^{\prime}(s) / G_{d}(s)$ is the implemented transfer function, and $F c(s)=F c_{n}(s) / F_{d}(s)$ is a corrective term.

Eq. (7) can be written as eq. (8) which is linear, where the unknowns are the coefficients of $G_{n}^{\prime}(s)$ and $F c_{n}(s)$.

$$
G_{n}^{\prime}(s) F_{n}(s)+F c_{n}(s) G_{d}(s)=G_{n}(s) F_{d}(s)
$$

The corrective term due to $F c(\mathrm{~s})$ can be replaced by its $\mathrm{Z}$ equivalent obtained by the classical formula

$$
F_{C z}(z)=\left(1-z^{-1}\right) Z_{T}\left\{L^{-1}\left[\frac{F_{c}(s)}{s} e^{-d s}\right]\right\}
$$

The term $F_{c}(z)$ will be replaced by its series expansion, as for a lowpass filter, its impulse response can be approximated by the first terms. The resulting modulator is the same as in Figure 7, where $G$ has been replaced by $G^{\prime}$, and $D(z)$ is replaced by $D(z)+F_{c}(z)$.

The adequate loop delay is calculated as in subsection $\mathrm{B}$, so that the phase of $G^{\prime}(s)$ at resonance is the same as the real filter.

\section{Simulation Results}

We consider in this part a delta sigma using a single BST resonator. This resonator is at this time under processing so we consider only transfer functions obtained by simulations. The admittance after compensation was given by Figure 5 . The sampling frequency $F s$ of the modulator is $3.2 \mathrm{GHz}$. The central frequency is equal to $3 F s / 4$. The amplifiers have been modeled by lowpass filters. As shown in Figure 3. the filter is composed with 3 active elements. Considering furthermore the filtering introduced by the impedances of the driver, we model all active elements by a fourth order lowpass filter. In this simulation, we suppose that the cutoff frequency is equal to the modulator sampling frequency $(3.2 \mathrm{GHz})$. This value was obtained by making preliminary simulations on the electrical models of the different active elements. Figure 7. shows the transfer function used during the modulator synthesis, the BST admittance, and real filter transfer function including the fourth order lowpass filtering. It can be seen that the lowpass function lowers the harmonic resonances, which is favorable in the case of a delta sigma modulator. Finally, in Figure 8. the Power Spectral Densities (PSD) of the output of the ideal modulator composed with a second-order resonator is compared to that of the 'real' modulator composed with a model of the real BST transmittance followed by a fourth order lowpass filter. It can be seen that the modulator is still stable with a noise spectrum comparable to that of the ideal modulator. It was also verified that the modulator behaves correctly when the resonance frequency changes from 2.42 to $2.48 \mathrm{Ghz}$.

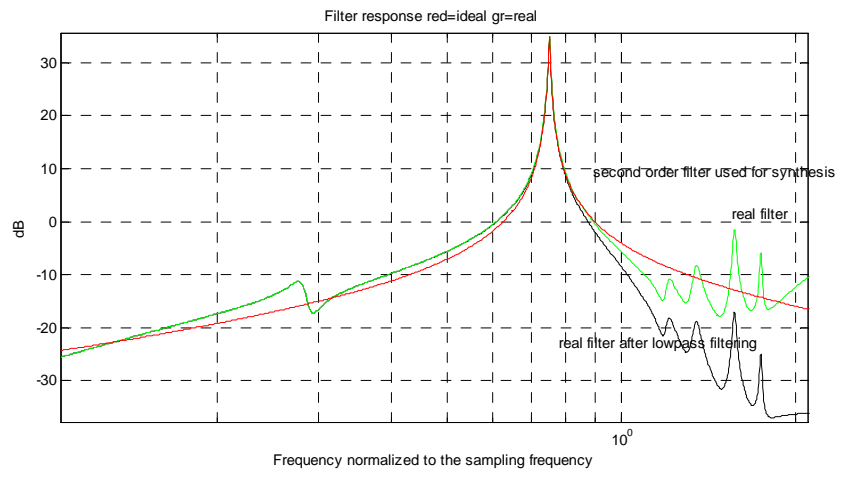

Figure 7. BST admitance and filter transfer function
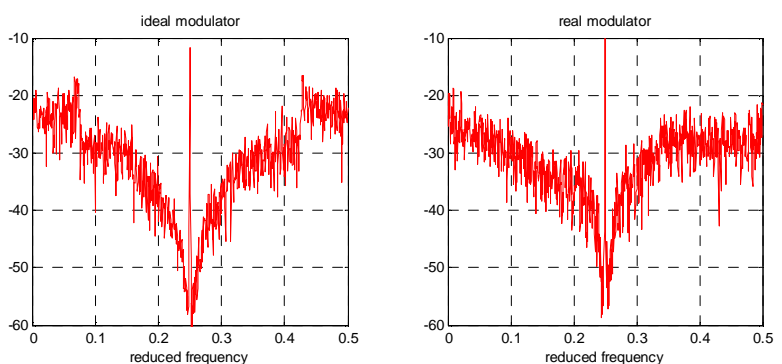

Figure 8. PSD of the ideal modulator compared with the real one

\section{CONCLUSION}

We have proposed in this paper a methodology for the high level design of BPCT $\Delta \Sigma$ modulators based on passive filters. This methodology uses an approximate model of the resonator around the main resonance to get the modulator coefficients. This principle was validated by simulations on a second order modulator. It can be transposed to higher order modulators, but the demonstration could not fit in this paper. A testchip will be designed when the models of BST elements are validated by measurements on real filters.

\section{REFERENCES}

[1] Yamada, T. ; Muralt, P. ; Tagantsev, A. ; Setter, N. ," Tunable thin film bulk acoustic wave resonator based on BaxSr1-xTiO3 thin film", IEEE Transactions on Ultrasonics, Ferroelectrics and Frequency Control, vol.57, issue 2, pp.379-385, 02/2010

[2] R.Yu,Y.P.Xu, "Bandpass sigma-delta modulator employing SAW resonator as loop filter", IEEE Tr. On Circuits \& Systems I, CASI-54, n4, pp. 723-735, April 2007

[3] ARTEMOS project : http://www.artemos.eu/

[4] Javidan, M., Benabes, P., « Band-Pass Continuous-Time Delta-Sigma Modulators Employing LWR Resonators », IEEE International Conference on Electronics, Circuits, and Systems (ICECS'08).

[5] R. Schreier, "Delta-sigma toolbox for MATLAB", Version 7.3, http://www.mathworks.com/matlabcentral/fileexchange/19-deltasigma-toolbox, Dec. 2011

[6] Benabes P., Gauthier A., Kielbasa R., « A multistage Closed-Loop Sigma-Delta Modulator (MSCL) », Journal of Analog Integrated Circuits and Signal Processing, vol 11, $\mathrm{n}^{\circ} 3$, pp. 195-204, novembre 1996.R. Nicole

[7] Benabes P., Keramat M., Kielbasa R., «Synthesis and analysis of sigma-delta modulators employing continuous-time filters », Analog Integrated Circuits and Signal Processing, $\mathrm{n}^{\circ} 23$, pp. 141-152, 2000. 